\begin{tabular}{|c|l|}
\hline Title & Fluorination of Ketones Using Iodotoluene Difluoride \\
\hline Author(s) & Sato, Saeko; Y oshida, Masanori; Hara, Shoji \\
\hline Citation & $\begin{array}{l}\text { Synthesis, 2005(15), 2602-2605 } \\
\text { https://doi.org/10.1055/52005-872105 }\end{array}$ \\
\hline Issue Date & 2005-10 \\
\hline Doc URL & http://hdl.handle.net/2115/650 \\
\hline Type & article (author version) \\
\hline File Information & 佐藤synth \\
\hline
\end{tabular}

Instructions for use 


\title{
Fluorination of Ketones Using Iodotoluene Difluoride
}

\author{
Saeko Sato, Masanori Yoshida, Shoji Hara* \\ Division of Molecular Chemistry, Graduate School of Engineering, Hokkaido University, Sapporo 060-8628, Japan \\ Fax: +81(11)7066556; \\ E-mail: hara@org-mc.eng.hokudai.ac.jp \\ Received:
}

Abstract: Fluorination of ketones was achieved by the reaction of silyl enol ethers with iodotoluene difluoride in the presence of $\mathrm{BF}_{3} \cdot \mathrm{OEt}_{2}$ and a $\mathrm{Et}_{3} \mathrm{~N} \cdot \mathrm{HF}$ complex.

Key words: fluorination, iodotoluene difluoride, silyl enol ether, ketone, hypervalent iodine

Introduction of a fluorine atom into the $\alpha$-position of carbonyl compounds is the most effective method for the synthesis of $\alpha$-fluorinated carbonyl compounds which have been used as important synthons in the preparation of more complex fluorine compounds.1,2 Though electrophilic fluorination reagents such as $\mathrm{N}$-fluoro reagents have been used for their synthesis, ${ }^{3}$ dangerous $F_{2}$ is necessary for their preparation, and such reagents are expensive even if they are commercially available. Recently we reported that the direct fluorination reaction of $\beta$-dicarbonyl compounds can be achieved using iodotoluene difluoride (ITDF) 4,5 which can be prepared in large quantity without $F_{2 .} 6,7$ However, application of ITDF is restricted to $\beta$-dicarbonyl compounds 4,5 or $\alpha$-sulfanylated carbonyl compounds, ${ }^{8-11}$ and ITDF is inert to monocarbonyl compounds. Previously, Tsushima et al. applied ITDF for the reaction with silyl enol ethers of steroidal ketones for the synthesis of steroidal fluoroketones. ${ }^{12}$ However, olefinic by-products were competitively formed and yields of the desired $\alpha$-fluoro ketones were low. During our continuous study of fluorination of carbonyl compounds using ITDF, we succeeded in improving the yield of fluorinated carbonyl compounds in the reaction of ITDF with silyl enol ethers.

When a silyl enol ether of acetophenone (1a) was allowed to react with ITDF at room temperature, it took 3 hours until the complete consumption of 1a, and acetophenone and its dimmer (5a) were obtained as main products. Under the conditions, the formation of an iodonium salt (2a), which must be a key intermediate for the fluorinated product (3a),12 seems to be slow and the generated iodonium salt $2 \mathrm{a}$ decomposed to acetophenone or reacted with the remaining $1 a$ to give $5 a$ (Scheme 1 ). Zhadnkin et al. reported that the iodonium salts can be stably prepared at lower temperature by the reaction of 1 with an iodoarene difluoride- $\mathrm{BF}_{3} \cdot \mathrm{OEt}_{2}$ complex. ${ }^{13}$ In order to make $2 \mathrm{a}$ at low temperature, $\mathrm{BF}_{3} \bullet \mathrm{OEt}_{2}$ was used with ITDF, and various fluoride 
sources were applied to accelerate the fluorination of $2 a$ (Table 1). Though la was consumed at $-78{ }^{\circ} \mathrm{C}$ in the presence of $\mathrm{BF}_{3} \cdot \mathrm{OEt}_{2}$, the addition of metal fluorides such as KF and CsF, which are only slightly soluble in an organic solvent, failed to improve the yields of $3 a$. On the other hand, the addition of $\mathrm{Et}_{3} \mathrm{~N} \cdot \mathrm{HF}$ complexes $\left(\mathrm{Et}_{3} \mathrm{~N} \cdot \mathrm{nHF}\right)$ could improve the results. When a commercially available $\mathrm{Et}_{3} \mathrm{~N} \cdot 3 \mathrm{HF}^{14,15}$ was used as the fluoride source, $3 a$ could be obtained in $71 \%$ yield with a little amount of $4 a(9 \%)$ and $5 \mathrm{a}(4 \%) . \mathrm{Et}_{3} \mathrm{~N} \cdot 2 \mathrm{HF}$ could slightly improve the result but $\mathrm{Et}_{3} \mathrm{~N} \cdot \mathrm{HF}, \mathrm{TBAF}$, and $\mathrm{Et}_{4} \mathrm{NF}$ were less effective than $\mathrm{Et}_{3} \mathrm{~N} \cdot 2 \mathrm{HF}$ or $\mathrm{Et}_{3} \mathrm{~N} \cdot 3 \mathrm{HF}$.

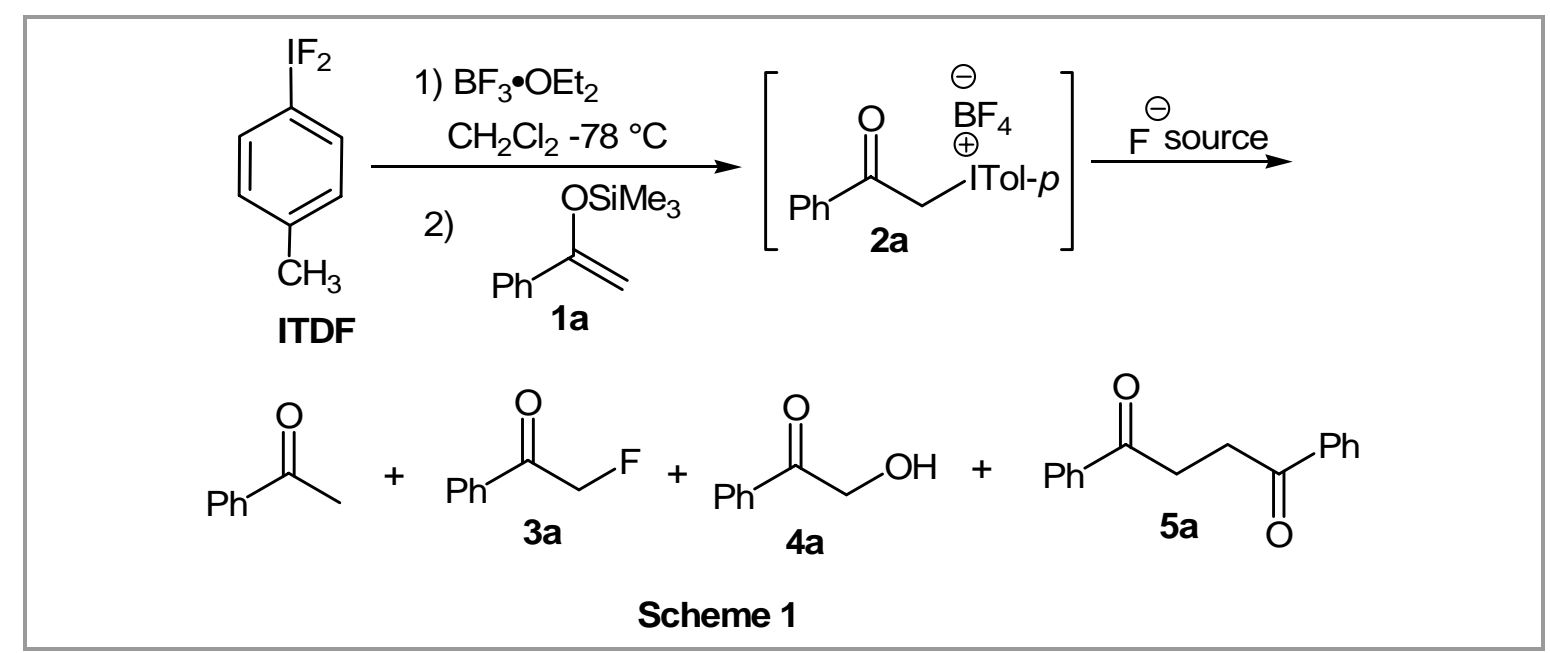

Table 1 Effect of Fluoride source on the Fluorination of $1 \mathbf{a}^{\mathrm{a}}$

\begin{tabular}{lccc}
\hline \multirow{2}{*}{ Fluoride source } & \multicolumn{3}{c}{ Yield (\%) $^{\mathrm{b}}$} \\
\hline $\mathrm{Et}_{3} \mathrm{~N} \cdot 3 \mathrm{H}$ & $\mathbf{4 a}$ & $\mathbf{5 a}$ \\
$\mathrm{Et}_{3} \mathrm{~N} \cdot 2 \mathrm{HF}$ & 71 & 9 & 4 \\
$\mathrm{Et}_{3} \mathrm{~N} \cdot \mathrm{HF}$ & 73 & 7 & 4 \\
$\mathrm{TBAF}$ & 40 & - & 10 \\
$\mathrm{Et}_{4} \mathrm{NF}$ & 25 & 9 & 3 \\
$\mathrm{CsF}^{\mathrm{c}}$ & 1 & 49 & 8 \\
$\mathrm{KF}^{\mathrm{c}}$ & - & 80 & 4 \\
\hline
\end{tabular}

a If otherwise not mentioned, $\mathrm{CH}_{2} \mathrm{Cl}_{2}$ was used as solvent.

b NMR yield based on 1a. ${ }^{\mathrm{c}} \mathrm{CH}_{3} \mathrm{CN}$ was used as solvent.

Under the conditions, silyl enol ethers of various acyclic and cyclic ketones could be converted to the corresponding $\alpha$-fluoroketones (3) (Table 2). 
Introduction of a fluorine atom to methyl (entry 4), methylene (entries 1,2,5-9), and even methyne carbon (entry 3) was also possible. When cyclic ketone silyl enol ethers were used (entries 6-9), the formation of an olefinic by-product was observed to an extent of $<5 \%$. Fluorinated analogs of steroids have been of great interest for the study of their metabolic mechanism, and 2-fluorocolesterols were synthesized from their enol esters by the reaction with electrophilic fluorinating regents. However, the products obtained by these methods are always $\alpha$-isomers which are more favorable both kinetically and thermodynamically, and it was difficult to synthesize the $\beta$-isomers. ${ }^{16-18}$ On the other hand, when silyl enol ether of cholestan-3-one (6) was treated with ITDF, the $\beta$-isomer of 2-fluorocholestan-3-one (7- $\beta$ ) was obtained as a main product (Scheme 2). This unusual stereoselectivity can be explained by the formation of the iodonium intermediate. ${ }^{12}$ ITDF attacks 6 from the less hindered $\alpha$-face to give the $\alpha$-isomer of the iodonium intermediate which reacts with a fluoride ion with inversion of stereochemistry to give the $7-\beta .19$

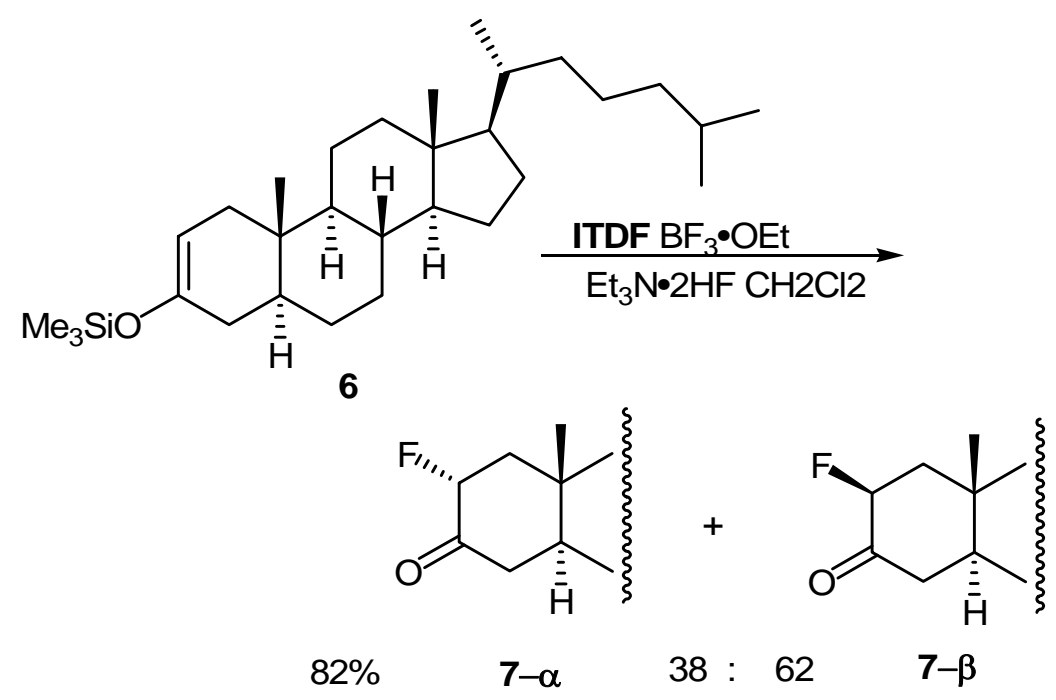

Scheme 2 
Table 2 Reaction of Sily Enol Ethers 1 with ITDFa

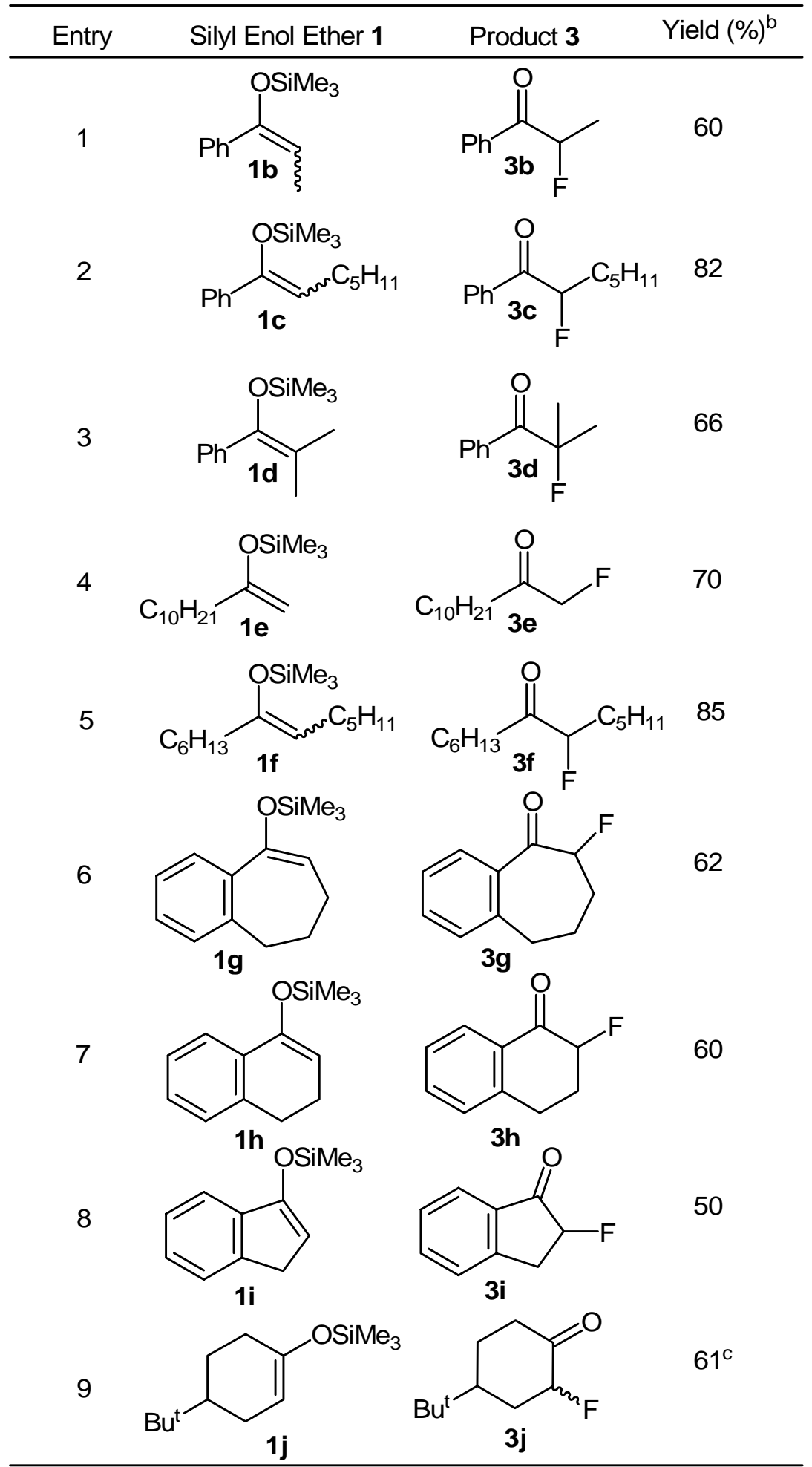

a The reactions were carried out in $\mathrm{CH}_{2} \mathrm{Cl}_{2}$ using ITDF, $\mathrm{BF}_{3} \cdot \mathrm{OEt}_{2}$, and $\mathrm{Et}_{3} \mathrm{~N} \cdot 2 \mathrm{HF}$ to 1 . b Isolated yields based on 1 and yields of olefinic byproducts were less than $5 \%$. c Ratio of cis : trans $=1: 1$ 
The IR spectra were recorded using a JASCO FT/IR-410. The ${ }^{1} \mathrm{H}$ NMR $(400 \mathrm{MHz}),{ }^{19} \mathrm{~F}$ NMR (376MHz), and ${ }^{13} \mathrm{C}$ NMR (100 MHz) spectra were recorded in $\mathrm{CDCl}_{3}$ on a JEOL JNM-A400II FT NMR and the chemical shift, $\delta$, are referred to TMS $\left({ }^{1} \mathrm{H},{ }^{13} \mathrm{C}\right)$ and $\mathrm{CFCl}_{3}\left({ }^{19} \mathrm{~F}\right)$, respectively. The EI-high-resolution mass spectra were measured on a JEOL JMS-700TZ, JMS-FABmate or JMS-HX110. Melting points are measured by Yanagimoto micro melting point apparatus and are uncorrected.

ITDF was prepared from iodotoluene as reported previously. ${ }^{7} \mathrm{BF}_{3} \bullet \mathrm{OEt}_{2}$ and $\mathrm{Et}_{3} \mathrm{~N} \cdot 3 \mathrm{HF}$ were purchased from Tokyo Kasei Co., Ltd., and distilled under $\mathrm{N}_{2}$ before use. $\mathrm{Et}_{3} \mathrm{~N} \cdot 2 \mathrm{HF}$ and $\mathrm{Et}_{3} \mathrm{~N} \cdot \mathrm{HF}$ were prepared by the addition of freshly distilled $\mathrm{Et}_{3} \mathrm{~N}$ to $\mathrm{Et}_{3} \mathrm{~N} \cdot 3 \mathrm{HF}$. The silyl enol esters 1 were prepared from the corresponding ketones according to a literature. ${ }^{20}$ Ketones were obtained from Tokyo Kasei Co., Ltd., and used without purification.

\section{Fluorination of Ketones 1 with Iodotoluene Difluoride; General Procedure}

To a $\mathrm{CH}_{2} \mathrm{Cl}_{2}$ solution $(10 \mathrm{~mL})$ of ITDF $(256 \mathrm{mg}, 1.0 \mathrm{mmol})$ in Teflon FEP vessel ${ }^{21}$ were added at $-78{ }^{\circ} \mathrm{C}, \mathrm{BF}_{3} \cdot \mathrm{OEt}_{2}(142 \mathrm{mg}, 1.0 \mathrm{mmol})$, and after $10 \mathrm{~min}$, silyl enol ether $\mathbf{1}(1.0 \mathrm{mmol})$. The reaction mixture was stirred at $-78{ }^{\circ} \mathrm{C}$ for $2 \mathrm{~h}$ and a $\mathrm{CH}_{2} \mathrm{Cl}_{2}$ solution $(5 \mathrm{~mL})$ of $\mathrm{Et}_{3} \mathrm{~N} \cdot 2 \mathrm{HF}(706 \mathrm{mg}$, $5.0 \mathrm{mmol}$ ) was added. After $1 \mathrm{~h}$, the mixture was poured into water and the separated aqueous layer was extracted with $\mathrm{CH}_{2} \mathrm{Cl}_{2}(2 \mathrm{X} 10 \mathrm{~mL})$. The combined organic layers were dried over $\mathrm{MgSO}_{4}$ and product 3 was obtained by column chromatography (silica gel/hexane- $\mathrm{Et}_{2} \mathrm{O}$ ).

\section{2-Fluoroacetophenone (3a)}

IR (neat): 2992, 1700, 1598, 1450, 1233, $1132 \mathrm{~cm}^{-1}$.

${ }^{1} \mathrm{H}$ NMR $\delta=5.55\left(\mathrm{~d}, J_{\mathrm{H}-\mathrm{F}}=46.8 \mathrm{~Hz}, 2 \mathrm{H}\right), 7.26-7.53(\mathrm{~m}, 2 \mathrm{H}), 7.62-7.66(\mathrm{~m}, 1 \mathrm{H}), 7.89-7.94(\mathrm{~m}, 2 \mathrm{H})$.

${ }^{19} \mathrm{~F}$ NMR $\delta=-231.4\left(\mathrm{t}, J_{\mathrm{H}-\mathrm{F}}=46.8 \mathrm{~Hz}, 1 \mathrm{~F}\right)$ [(lit. $\left.{ }^{22}-230.3(\mathrm{t}, J=47 \mathrm{~Hz})\right]$.

\section{2-Fluoro-1-phenyl-1-propanone (3b)}

IR (neat): 2955, 2932, 2860, 1699, 1597, $1449 \mathrm{~cm}^{-1}$.

${ }^{1} \mathrm{H}$ NMR: $\delta=1.64\left(\mathrm{dm}, J_{\mathrm{H}-\mathrm{F}}=38.6 \mathrm{~Hz}, 3 \mathrm{H}\right), 5.70\left(\mathrm{dq}, J_{\mathrm{H}-\mathrm{F}}=48.8, J_{\mathrm{H}-\mathrm{H}}=5.6 \mathrm{~Hz}, 1 \mathrm{H}\right), 7.48-7.52(\mathrm{~m}$, 2H), 7.60-7.64 (m, 1H), 7.96-7.99 (m, 2H).

${ }^{19}$ F NMR: $\delta=-181.95$ to -182.27 (m, 1F) (lit. $\left.{ }^{22}-180.5\right)$.

\section{2-Fluoro-1-phenyl-1-heptanone (3c)}

IR (neat): 2955, 2932, 2860, 1699, 1597, $1449 \mathrm{~cm}^{-1}$.

${ }^{1} \mathrm{H}$ NMR: $\delta=0.89$ (t, $\left.J_{\mathrm{H} . \mathrm{H}}=7.3 \mathrm{~Hz}, 3 \mathrm{H}\right), 1.30-1.38(\mathrm{~m}, 4 \mathrm{H}), 1.50-1.57(\mathrm{~m}, 2 \mathrm{H}), 1.90-2.02(\mathrm{~m}, 2 \mathrm{H})$, $5.57\left(\mathrm{dm}, J_{\mathrm{H} . \mathrm{F}}=49.5 \mathrm{~Hz}, 1 \mathrm{H}\right), 7.47-7.97(\mathrm{~m}, 5 \mathrm{H})$.

${ }^{19}$ F NMR: $\delta=-190.54--190.27(\mathrm{~m}, 1 \mathrm{~F})$.

${ }^{13}$ C NMR: $\delta=14.01,22.47,24.54$ (d, $\left.J_{\mathrm{C} . \mathrm{F}}=3.3 \mathrm{~Hz}\right), 31.41,32.83$ (d, $\left.J_{\mathrm{C} . \mathrm{F}}=20.7 \mathrm{~Hz}\right), 93.97\left(\mathrm{~d}, J_{\mathrm{C} . \mathrm{F}}=\right.$ $182.8 \mathrm{~Hz}), 128.80(2 \mathrm{C}), 128.91$ (d, $J_{\mathrm{C} . \mathrm{F}}=3.3 \mathrm{~Hz}$ ), $133.78(2 \mathrm{C}), 134.42,197.03$ (d, $J_{\mathrm{C} . \mathrm{F}}=19.8 \mathrm{~Hz}$ ).

HRMS (EI): m/z calcd for $\mathrm{C}_{13} \mathrm{H}_{17} \mathrm{OF}$ : 208.1250; found: 208.1257 .

\section{2-Fluoro-2-methyl-1-phenyl-1-propanone (3d)}

IR (neat): 2988, 1685, $1179 \mathrm{~cm}^{-1}$.

${ }^{1} \mathrm{H}$ NMR: $\delta=1.70$ (d, $\left.J_{\mathrm{H} . \mathrm{F}}=21.5 \mathrm{~Hz}, 6 \mathrm{H}\right), 7.44-7.48(\mathrm{~m}, 2 \mathrm{H}), 7.55-7.58(\mathrm{~m}, 1 \mathrm{H}), 8.06-8.08(\mathrm{~m}, 2 \mathrm{H})$.

${ }^{19}$ F NMR: $\delta=-144.32$ to -143.98 (m, 1F) (lit. $\left.{ }^{22}-142.1\right)$.

\section{1-Fluoro-2-dodecanone (3e)}


IR (neat): 2925, 2855, 1728, 1465, 1047.

${ }^{1} \mathrm{H}$ NMR: $\delta=0.88\left(\mathrm{t}, J_{\mathrm{H} . \mathrm{F}}=7.0 \mathrm{~Hz}, 3 \mathrm{H}\right), 1.26-1.30(\mathrm{~m}, 14 \mathrm{H}), 1.56-1.65(\mathrm{~m}, 2 \mathrm{H}), 2.54\left(\mathrm{td}, J_{\mathrm{H} . \mathrm{H}}=7.4\right.$, $\left.J_{\mathrm{H} . \mathrm{F}}=2.9 \mathrm{~Hz}, 2 \mathrm{H}\right), 4.80\left(\mathrm{~d}, J_{\mathrm{H} . \mathrm{F}}=47.8 \mathrm{~Hz}, 2 \mathrm{H}\right)$.

${ }^{19}$ F NMR: $\delta=-228.01\left(\mathrm{t}, J_{\mathrm{H} . \mathrm{F}}=47.8 \mathrm{~Hz}, 1 \mathrm{~F}\right)$.

${ }^{13}$ C NMR: $\delta=14.12,22.69,22.76,29.15,29.31,29.35,29.45,29.55,31.90,38.31,84.97$ (d, $J_{\text {C.F }}=$ $184.4 \mathrm{~Hz}), 207.29$ (d, $J_{\mathrm{C} . \mathrm{F}}=19.0 \mathrm{~Hz}$ ).

HRMS (EI): m/z calcd for $\mathrm{C}_{12} \mathrm{H}_{23}$ FO: 202.1741; found: 202.1737 .

\section{6-Fluoro-7-tridecanone (3f)}

IR (neat): 2956, 2932, 1699, 1597, $1449 \mathrm{~cm}^{-1}$.

${ }^{1} \mathrm{H}$ NMR: $\delta=0.87-0.91(\mathrm{~m}, 6 \mathrm{H}), 1.28-1.37(\mathrm{~m}, 10 \mathrm{H}), 1.40-1.48(\mathrm{~m}, 2 \mathrm{H}), 1.54-1.61(\mathrm{~m}, 2 \mathrm{H})$, $1.70-1.86(\mathrm{~m}, 2 \mathrm{H}), 2.53-2.64(\mathrm{~m}, 2 \mathrm{H}), 4.71\left(\mathrm{dm}, J_{\mathrm{H} . \mathrm{F}}=50.4 \mathrm{~Hz}, 1 \mathrm{H}\right)$.

${ }^{19} \mathrm{~F}$ NMR: $\delta=-192.64$ to -192.35 (m, $\left.1 \mathrm{~F}\right)$.

${ }^{13}$ C NMR: $\delta=13.97,14.03,22.44$ (d, $J_{\text {C.F }}=10.8 \mathrm{~Hz}$ ), 22.64, 24.21 (d, $\left.J_{\mathrm{C} . \mathrm{F}}=2.5 \mathrm{~Hz}\right), 28.84,31.34$, 31.58, 31.93, 32.14, 38.04, 96.09 (d, $\left.J_{\mathrm{C} . \mathrm{F}}=183.6 \mathrm{~Hz}\right), 210.54$ (d, $\left.J_{\mathrm{C} . \mathrm{F}}=24.8 \mathrm{~Hz}\right)$.

HRMS (EI): $\mathrm{m} / \mathrm{z}$ calcd for $\mathrm{C}_{13} \mathrm{H}_{25} \mathrm{OF}$ : 216.1890; found: 216.1890.

\section{6-Fluoro-6,7,8,9-tetrahydrobenzocyclohepten-5-one (3g)}

IR (neat): 2943, 1696, 1599, $1449 \mathrm{~cm}^{-1}$.

${ }^{1} \mathrm{H}$ NMR: $\delta=1.89-2.19(\mathrm{~m}, 3 \mathrm{H}), 2.29-2.43(\mathrm{~m}, 1 \mathrm{H}), 2.92-3.08(\mathrm{~m}, 2 \mathrm{H}), 5.24\left(\mathrm{dm}, J_{\mathrm{H} . \mathrm{F}}=48.8 \mathrm{~Hz}\right.$, $1 \mathrm{H}), 7.22-7.26(\mathrm{~m}, 1 \mathrm{H}), 7.31-7.35(\mathrm{~m}, 1 \mathrm{H}), 7.42-7.46(\mathrm{~m}, 1 \mathrm{H}), 7.77$ (d, $\left.J_{\mathrm{H}, \mathrm{F}}=7.8 \mathrm{~Hz}, 1 \mathrm{H}\right)$.

${ }^{19}$ F NMR: $\delta=-183.3$ to -183.15 (m, 1F) $\left(\right.$ lit. $\left.{ }^{23}-183\right)$.

\section{2-Fluoro-1-tetralone (3h)}

White solid; Mp $34{ }^{\circ} \mathrm{C}$ (lit. ${ }^{23} 38-40{ }^{\circ} \mathrm{C}$ ).

IR (KBr): 2938, 2898, 1707, 1602, 1271, $1227 \mathrm{~cm}^{-1}$.

${ }^{1} \mathrm{H}$ NMR: $\delta=2.31-2.43(\mathrm{~m}, 1 \mathrm{H}), 2.55-2.63(\mathrm{~m}, 1 \mathrm{H}), 3.13-3.16(\mathrm{~m}, 2 \mathrm{H}), 5.14\left(\mathrm{dm}, J_{\mathrm{H} . \mathrm{F}}=48.1 \mathrm{~Hz}\right.$, $1 \mathrm{H}), 7.26-7.55(\mathrm{~m}, 3 \mathrm{H}), 8.08$ (d, $\left.J_{\mathrm{H} . \mathrm{F}}=7.8 \mathrm{~Hz}, 1 \mathrm{H}\right)$.

${ }^{19}$ F NMR: $\delta=-190.99\left(\mathrm{dm}, J_{\mathrm{H} . \mathrm{F}}=48.2,1 \mathrm{~F}\right)\left[\left(\mathrm{lit} .{ }^{23}-192\left(\mathrm{dm}, J_{\mathrm{H} . \mathrm{F}}=47.5 \mathrm{~Hz}\right)\right]\right.$.

\section{2-Fluoroindan-1-one (3i)}

White solid; Mp 56-58 ${ }^{\circ} \mathrm{C}$ (lit. ${ }^{23} 54-56^{\circ} \mathrm{C}$ ).

IR (KBr): 2924, 1719, 1607, 1465, $1087 \mathrm{~cm}^{-1}$.

${ }^{1} \mathrm{H}$ NMR: $\delta=3.19-3.30(\mathrm{~m}, 1 \mathrm{H}), 3.60-3.68(\mathrm{~m}, 1 \mathrm{H}), 5.27\left(\mathrm{dm}, J_{\mathrm{H} . \mathrm{F}}=51.0 \mathrm{~Hz}, 1 \mathrm{H}\right), 7.43-7.48(\mathrm{~m}$, $2 \mathrm{H}), 7.66-7.70(\mathrm{~m}, 1 \mathrm{H}), 7.81(\mathrm{~d}, J=7.6 \mathrm{~Hz}, 1 \mathrm{H})$.

${ }^{19}$ F NMR: $\delta=-194.17$ (ddd, $J_{\text {H.F }}=51.0,23.2,7.4 \mathrm{~Hz}, 1 \mathrm{~F}$ ) [(lit. ${ }^{23}-192.5$ (ddd, $J_{\mathrm{H} . \mathrm{F}}=50,22.5,9 \mathrm{~Hz}$ )].

\section{cis-2-Fluoro-4-tert-butylcyclohexanone (cis-3j)}

White solid; Mp $37^{\circ} \mathrm{C}$ (lit. ${ }^{16} 40^{\circ} \mathrm{C}$ ).

IR (KBr): 2962, 1737, $1368 \mathrm{~cm}^{-1}$.

${ }^{1} \mathrm{H}$ NMR: $\delta=0.95$ (s, 9H), 1.39-1.50 (m, 1H), 1.54-1.69 (m, 2H), 2.07-2.18 (m, 1H), 2.28-2.37 (m, $1 \mathrm{H}), 2.49-2.56(\mathrm{~m}, 2 \mathrm{H}), 4.93\left(\mathrm{dm}, J_{\mathrm{H} . \mathrm{F}}=48.5 \mathrm{~Hz}, 1 \mathrm{H}\right)$.

${ }^{19}$ F NMR: $\delta=-188.62\left(\mathrm{dm}, J_{\mathrm{H} . \mathrm{F}}=48.2 \mathrm{~Hz}, 1 \mathrm{~F}\right)$. 
trans-2-Fluoro-4-tert-butylcyclohexanone (trans-3j)

White solid; Mp 69-71 ${ }^{\circ} \mathrm{C}$ (lit. ${ }^{16} 74^{\circ} \mathrm{C}$ ).

IR (KBr): 2956, 2871, 1722, $1365 \mathrm{~cm}^{-1}$.

${ }^{1}$ H NMR: $\delta=0.92(\mathrm{~s}, 9 \mathrm{H}), 1.42-1.70(\mathrm{~m}, 2 \mathrm{H}), 1.84-1.92(\mathrm{~m}, 1 \mathrm{H}), 2.10-2.19(\mathrm{~m}, 1 \mathrm{H}), 2.35-2.45(\mathrm{~m}$, $2 \mathrm{H}), 2.76-2.86(\mathrm{~m}, 1 \mathrm{H}), 4.67\left(\mathrm{dm}, J_{\mathrm{H} . \mathrm{F}}=46.8 \mathrm{~Hz}, 1 \mathrm{H}\right)$.

${ }^{19}$ F NMR: $\delta=-186.28$ to $-185.98(\mathrm{~m}, 1 \mathrm{~F})$.

\section{2 $\alpha$-Fluorocholestan-3-one (7- $\alpha)$}

White solid. Mp $161-163{ }^{\circ} \mathrm{C}$ (lit. ${ }^{17} 168-169{ }^{\circ} \mathrm{C}$ )

IR (KBr): 2939, 2865, 1735, 1467, $1382 \mathrm{~cm}^{-1}$.

${ }^{1} \mathrm{H}$ NMR: $\delta=0.85-2.03(\mathrm{~m}, 41 \mathrm{H}), 2.21-2.53(\mathrm{~m}, 3 \mathrm{H}), 4.98\left(\mathrm{dm}, J_{\mathrm{H} . \mathrm{F}}=48.1 \mathrm{~Hz}, 1 \mathrm{H}\right)$.

${ }^{19}$ F NMR: $\delta=-194.67$ (dm, $J_{\text {H.F }}=48.1 \mathrm{~Hz}, 1 \mathrm{~F}$ ) (lit. ${ }^{17}$-194.48).

\section{2 $\beta$-Fluorocholestan-3-one (7- $\beta$ )}

White solid; Mp $85-87^{\circ} \mathrm{C}$.

IR (KBr): 2932, 2861, 1735, 1467, $1382 \mathrm{~cm}^{-1}$.

${ }^{1} \mathrm{H}$ NMR: $\delta=0.85-1.87(\mathrm{~m}, 40 \mathrm{H}), 1.97-2.02(\mathrm{~m}, 1 \mathrm{H}), 2.16-2.26(\mathrm{~m}, 2 \mathrm{H}), 2.59-2.68(\mathrm{~m}, 1 \mathrm{H}), 4.79(\mathrm{dt}$, $\left.J_{\mathrm{H} . \mathrm{F}}=49.8, J_{\mathrm{H} . \mathrm{H}}=4.6 \mathrm{~Hz}, 1 \mathrm{H}\right)$.

${ }^{19}$ F NMR: $\delta=-183.72$ to $-184.00(\mathrm{~m}, 1 \mathrm{~F})$.

${ }^{13} \mathrm{C}$ NMR: $\delta=12.03,13.82,13.86,18.64,21.51,22.55,22.80,23.80,24.14,27.99,28.19,28.64$, 31.47, 34.91, 35.74, 35.99, 36.11, 39.48, 39.81, 41.69, 44.90 (d, $\left.J_{\mathrm{C} . \mathrm{F}}=19.0 \mathrm{~Hz}\right), 45.84,54.68,56.10$, 56.21, $92.33\left(\mathrm{~d}, J_{\mathrm{C} . \mathrm{F}}=180.3 \mathrm{~Hz}\right), 206.90\left(\mathrm{~d}, J_{\mathrm{C} . \mathrm{F}}=18.2 \mathrm{~Hz}\right)$.

HRMS (EI): m/z calcd for $\mathrm{C}_{27} \mathrm{H}_{45} \mathrm{FO}: 404.3454$; found: 404.3453.

\section{References}

(1) Rozen, S.; Filler, R. Tetrahedron 1985, 41, 1111.

(2) Welch, J. T.; Eswarakrishnan, S. Fluorine in Bioorganic Chemistry; Wiley: New York, 1991.

(3) For a review article of electrophilic fluorination, see: Taylor, S. D.; Kotoris, C. C.; Hum, G. Tetrahedron 1999, 55, 12431.

(4) Hara, S.; Sekiguchi, M.; Ohmori, A.; Fukuhara, T.; Yoneda, N. Chem. Commun. 1996, 1899.

(5) Yoshida, M.; Fujikawa, K.; Sato, S.; Hara, S. Arkivoc 2003, 36.

(6) Carpenter, W. J. Org. Chem. 1966, 31, 2688.

(7) Sawaguchi, M.; Ayuba, S.; Hara, S. Synthesis 2002, 1802.

(8) Greaney, M. F.; Motherwell, W. B. Tetrahedron Lett. 2000, 41, 4463.

(9) Greaney, M. F.; Motherwell, W. B. Tetrahedron Lett. 2000, 41, 4467.

(10) Greaney, M. F.; Motherwell, W. B. Tocher, D. A. Tetrahedron Lett. 2001, 42, 8523.

(11) Motherwell, W. B.; Greaney, M. F.; Tocher, D. A. J. Chem. Soc., Perkin Trans. 1 2002, 2809.

(12) Tsushima, T.; Kawada, K.; Tsuji, T. Tetrahedron Lett. 1982, 23, 1165.

(13) They obtained dimmer 5 in good yields by this method, see: Zefirov, N. S.; Samoniya, N. S. Kutateladze, T. G.; Zhdankin, V. V. Zh. Org. Khim. 1991, 27, 220; Chem. Abstr. 1991, 115, 9164.

(14) McClinton, M. A. Aldrichimica Acta 1995, 28, 75.

(15) Haufe, G. J. Prakt. Chem. 1996, 338, 99.

(16) Rozen, S.; Menahem, Y. J. Fluorine Chem. 1980, 16, 19.

(17) Thomas, M. G.; Suckling, C. J.; Pitt, A. R.; Suckling, K. E. J. Chem. Soc., Perkin Trans. 1 1999, 3191.

(18) Stavber, S.; Jereb, M.; Zupan, M. Synthesis 2002, 2609.

(19) An equilibrium between two isomers of 7 was not observed under the reaction conditions.

(20) Eames, J.; Coumbarides, G. S.; Suggate, M. J.; Weerasooriya, N. Eur. J. Org. Chem. 2003, 634.

(21) A centrifuge tube with a screw cup made of Teflon FEP, a copolymer of TFE and hexafluoropropylene, was used.

(22) Cousseau, J.; Albert, P. J. Org. Chem. 1989, 54, 5380.

(23) Stavber, S.; Sket, B.; Zajc, B.; Zupan, M. Tetrahedron 1989, 45, 6003. 